\title{
INTERACTIVE SEQUENCES MODULATE THE SELECTION OF EXPRESSIVE FORMS IN CROSS-SIGNING
}

\author{
Kang-Suk Buyn" ${ }^{* 1}$, Connie de Vos ${ }^{2}$, Seán G. Roberts ${ }^{3}$, and Stephen C. Levinson ${ }^{1}$ \\ *Corresponding Author: Kang-Suk.Byun@mpi.nl \\ ${ }^{1}$ Language and Cognition Department, Max Planck Institute for Psycholinguistics, Nijmegen, \\ Netherlands \\ ${ }^{2}$ Center for Language Studies, Radboud University Nijmegen, Netherlands \\ ${ }^{3}$ excd.lab, University of Bristol, UK
}

This study looks at how linguistic conventions arise in the context of face-toface, real-time interaction. This topic is difficult to study because initial contact events for most languages happened long ago. Two approaches try to tackle this. The first is experimental semiotics: putting people in a situation where they need to improvise a new communication system. Studies have considered the relative role of different biases in this process (Tamariz, et al., 2014) such as frequency bias (where the more frequent signs prevail), content bias (where the more iconically - motivated, and/or easily - articulated form is selected), and coordination bias (where participants attempt to match each other's usage). However, these experiments typically involve an artificial language or a restricted or unfamiliar communication medium which are used by participants who already share a common language and culture. This limits the ecological validity of these experiments, especially relating to face-to-face interaction.

Another approach has been to study the emergence of new signed languages which emerge spontaneously from scratch, allowing the study of the formation processes of modern human languages in real life. This process has been welldocumented in the case of Nicaraguan Sign Language, which emerged over the course of several decades in a deaf school (Senghas \& Coppola, 2001), as well as in multiple 'deaf villages' where a local sign language has emerged from the interaction of deaf and hearing community members (Meir et al. 2010). Lesser-known instances of de novo signed communication arises between deaf and hearing nonsigners (trans-languaging), and among deaf signers who do not know a common written or signed language (cross-signing, Kusters et al. 2017; Buyn et al., 2017). Cross-signing is of particular interest as it creates a real-time pressure to establish a shared communicative repertoire. However, these studies are often not designed to be experimentally controlled, and rarely capture the very first period of the emergence of a signed language.

In this study we combine the control of experimental semiotics with the eco- 
logical validity of cross-signing in order to assess the relative contribution of different biases to the emergence of a shared lexicon. We recreated the context for cross-signing to emerge in a lab by flying deaf individuals from Nepal, Jordan and Indonesia to India and documenting their first encounters. This resulted in a 320-minute video corpus between dyads of signers of Nepali Sign Language, Indian Sign Language, Jordanian Sign Language and Indonesian Sign Language (these languages are mutually unintelligible and none of the signers knew any of the other languages). Recordings took place at the first meeting, after one week of living together, and after three weeks and included both spontaneous conversations and structured communication tasks, including the spot-the-differences task reported here. In this task, two signers were given a cartoon image each which differed only in the way certain objects were coloured. They could not see their partner's image. Their task was to identify the differences in colour by communicating spontaneously face-to-face. We coded and examined the various colour expressions exploited by the participants. The pairs repeated the experiment with new images after 1 week and after 3 weeks.

Participants initially used a range of strategies, including pointing, articulating signs for common objects with that colour (e.g. referring to a common iconic sign for 'tree' and pointing to the base to mean 'brown'), and their own native variants. However, after three weeks a consensus has been formed. For example, everyone uses the Indian signer's variant for 'green' and the Nepali signer's improvised 'tree-trunk' variant for 'brown'. Sequential analyses indicate that signers continuously assess the relative ease with which their forms might be understood, and adopt interactional strategies (e.g. try-marking, repair sequences) to manage communicative difficulties that arise. Mixed effects modelling was used to analyse which factors promoted the selection of particular signs. There were significant effects for frequency, content and coordination biases, supporting the findings of previous experimental studies, but the interactional context in which a form was used also mattered. This ability to study the very early stages of language emergence in a real yet controlled environment is an exciting addition to language evolution research approaches.

\section{Acknowledgements}

SGR supported by a Leverhulme early career fellowship to SGR (ECF-2016-435) and an European Research Council Advanced Grant No. 269484 INTERACT to SCL.

\section{References}

Byun, K.-S., De Vos, C., Bradford, A., Zeshan, U., \& Levinson, S. C. (2017). First encounters: Repair sequences in cross-signing. Topics in Cognitive Science, doi:10.1111/tops.12303. 
Kusters, A., Spotti, M., Swanwick, R., \& Tapio, E. (2017). Beyond languages, beyond modalities: transforming the study of semiotic repertoires. International Journal of Multilingualism, 1-14.

Meir, I., Sandler, W., Padden, C., Aronoff, M., et al.. (2010). Emerging sign languages. Oxford handbook of deaf studies, language, and education, 2, 267-280.

Senghas, A., \& Coppola, M. (2001). Children creating language: How nicaraguan sign language acquired a spatial grammar. Psychological science, 12(4), 323-328.

Tamariz, M., Ellison, T. M., Barr, D. J., \& Fay, N. (2014). Cultural selection drives the evolution of human communication systems. Proceedings of the Royal Society of London B: Biological Sciences, 281(1788), 20140488. 